\title{
Avaliação das dimensões orbitárias de coelhos após enucleação e evisceração
}

\author{
Rabbitorbitalmeasure evaluations after enucleation and evisceration
}

\author{
Claudia Akemi Shiratori ${ }^{1}$ \\ Silvana Artioli Schellini ${ }^{2}$ \\ Seizo Yamashita ${ }^{3}$ \\ Carlos Roberto Padovani ${ }^{4}$ \\ Romualdo Rossa ${ }^{5}$
}

Trabalho realizado na Faculdade de Medicina de Botucatu - UNESP. Botucatu (SP).

Pós-graduanda do Departamento de OFT/ORL/CCP Faculdade de Medicina de Botucatu - UNESP. Botucatu (SP).

2 Professora-Adjunta do Departamento de OFT/ORL/ CCP - UNESP. Botucatu (SP).

${ }^{3}$ Professor Assistente do Departamento de Radiologia UNESP. Botucatu (SP).

${ }^{4}$ Professor Titular do Departamento de Bioestatística do Instituto de Biociências - UNESP. Botucatu (SP).

${ }^{5}$ In Memorium.

Endereço para correspondência: Faculdade de Medicina de Botucatu-UNESP. Departamento de OFT/ORL/ CCP. Distrito Rubião Júnior, s/n - Botucatu (SP) CEP 18610-000

E-mail: sartioli@fmb.unesp.br

Recebido para publicação em 15.04.2004

Versão revisada recebida em 15.12.2004

Aprovação em 17.12.2004

Nota Editorial: Após concluída a análise do artigo sob sigilo editorial e com a anuência do Dr. Mário Luiz Ribeiro Monteiro sobre a divulgação de seu nome como revisor dele, agradecemos sua participação nesse processo.

\begin{tabular}{|l|}
\hline \multicolumn{1}{|c|}{ RESUMO } \\
\hline Objetivo: Avaliaro volume orbitário de coelhos recém-nascidos, enucleados \\
ou eviscerados, sem ou com reposição do volume orbitário por esferas de \\
polietileno poroso de $10 \mathrm{~mm}$, usando a tomografia computadorizada, o teste \\
de deslocamento de água e a medida da abertura orbitária por Image-J. \\
Métodos: Estudo experimental, usando 48 coelhos albinos, com idade de \\
42 dias, submetidos à evisceração ou enucleação, com implante de esfera \\
de polietileno poroso de 10 mm de diâmetro ou sem implante. O lado \\
esquerdo não foi submetido à cirurgia e foi usado como controle. Após 1, \\
3 e 6 meses, foram sacrificados quatro animais de cada grupo. Os crânios \\
foram preparados para estudo do osso seco por maceração da peça, e \\
analisados em relação ao volume orbitário por tomografia computadorizada, \\
teste de deslocamento de água e avaliação da abertura orbitária usando o \\
software Image-J. Resultados: O volume das órbitas enucleadas ou evis- \\
ceradas, com ou sem o uso do implante não apresentaram diferenças \\
estatisticamente significativas (p<0,05) entre si, quando avaliadas por \\
tomografia computadorizada, pelo teste de deslocamento de água e pelo \\
Image-J. Conclusão: O desenvolvimento orbitário de coelhos com idade \\
superior a 42 dias de vida não se altera quando se opta pela enucleação ou \\
pela evisceração, ou quando se utiliza ou não implantes orbitários. O teste \\
de deslocamento de água apresentou resultados semelhantes aos for- \\
necidos pela tomografia, sugerindo-se sua utilização em experimentação \\
científica.
\end{tabular}

Descritores: Enucleação ocular; Exenteração orbitária; Órbita/crescimento \& desenvolvimento; Tomografia computadorizada por raios X; Coelhos

\section{INTRODUÇÃOO}

A perda do olho ou de seu conteúdo pode provocar defeitos estéticos importantes, principalmente quando ocorre em fase precoce, durante o período de crescimento. Além disso, a anoftalmia é estigmatizante e psicologicamente comprometedora.

O reconhecimento da influência do conteúdo orbitário para o desenvolvimento ósseo da órbita e de estruturas adjacentes, levou ao desenvolvimento de técnicas, preconizando o uso de implantes intra-orbitários para reposição do volume deficiente, usados desde o século $\mathrm{XIX}^{(1)}$.

Porém, avaliar o volume orbitário a ser reposto não é tarefa fácil. Vários métodos já foram descritos para analisar o volume da órbita, havendo os métodos diretos, que analisam a órbita livre de seu conteúdo, ou métodos indiretos, que se baseiam em exames de imagem.

Entre os métodos diretos, existem os métodos de impressão ("imprin- 
ting") ou preenchimento, usando-se materiais como o sal, areia, borracha, cujo peso é dividido pelo coeficiente de gravidade específica do respectivo material, obtendo-se a medida do volume.

Os métodos indiretos inicialmente utilizavam radiografia e ultra-sonografia e, mais recentemente, a tomografia computadorizada e a ressonância magnética ${ }^{(2-4)}$. A tomografia computadorizada é um método que possibilita reconstruções tridimensionais, sendo eficiente para avaliar as estruturas orbitárias, principalmente quanto à conformação e dimensões ${ }^{(5-8)}$. A limitação ao seu uso é o preço, o que a torna um exame nem sempre disponível, principalmente para pesquisas.

A quantidade de conteúdo orbitário que se perde depende da causa que levou a perda do olho, da técnica cirúrgica empregada, da fase da vida em que se dá a perda do olho, além de outras variáveis que influenciam o crescimento da órbita. Para compreender melhor a influência destes fatores sobre o desenvolvimento orbitário, medidas objetivas de análise devem ser procuradas.

Assim, o objetivo deste estudo foi avaliar o volume orbitário de coelhos recém-desmamados, submetidos à cirurgia de enucleação ou de evisceração, com ou sem a reposição do volume orbitário por meio de esferas de polietileno poroso, usando-se métodos diretos e indiretos.

\section{MÉTODOS}

Este estudo foi experimental, prospectivo, casualizado, empregando-se 48 coelhos albinos da raça Norfolk, linhagem Botucatu, fornecidos pelo Biotério Central - Campus de Botucatu - UNESP, apresentando peso médio de $1000 \mathrm{~g}$ e idade de 42 dias. O protocolo foi submetido ao Comitê de Ética em Pesquisa em Animais desta Unidade, tendo sido aprovado.

Vinte e quatro animais tiveram os olhos direitos enucleados (EN) e outros 24, eviscerados (EV), sendo subdivididos em 2 grupos: com (CI) e sem (SI) a colocação de esfera de polietileno poroso (PP) de $10 \mathrm{~mm}$ de diâmetro (Mackron Biotecnologia, São
Paulo, Brasil). A órbita contralateral não sofreu cirurgia, tendo sido considerada como o grupo normal (GN). Quatro animais de cada grupo foram sacrificados em 3 momentos experimentais: 1 (M1), 3 (M2) e 6 (M3) meses após a cirurgia.

A avaliação das dimensões orbitárias foi feita por tomografia computadorizada e pelo teste de deslocamento de água, com o crânio preparado para estudo do osso seco (Figura 1).

Foram analisadas as imagens tomográficas obtidas de cortes no plano axial (perpendicular ao maior eixo corporal), com incremento de $2 \mathrm{~mm}$ e espessura de $2 \mathrm{~mm}$ (tomógrafo General Eletric (GE), modelo Sytec, software 4000 - i), realizando-se medidas lineares, com as seguintes estruturas de referência (Figura 2):

1) Distância do forame óptico até a reborda orbitária inferior $\left(\mathrm{D}_{1}\right)$;

2) Distância do processo supra-orbital anterior ao processo alveolar maxilar $\left(\mathrm{D}_{2}\right)$;

3) Distância da incisura do osso lacrimal até a reborda orbitária inferior, na região do início do arco zigomático (L), em imagem obtida por reformatação dos cortes obtidos axialmente.

Utilizando-se as medidas 1, 2 e 3, foi feito o cálculo estimado do volume da órbita, aplicando-se a seguinte fórmula matemática:

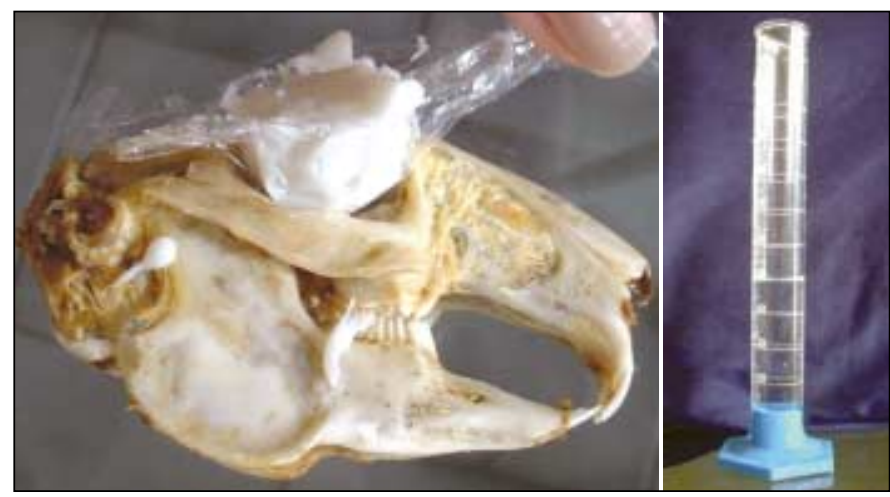

Figura 1 - Cranio seco. Demonstração da obtenção do molde a se colocado no cilindro graduado para o teste do deslocamento de água
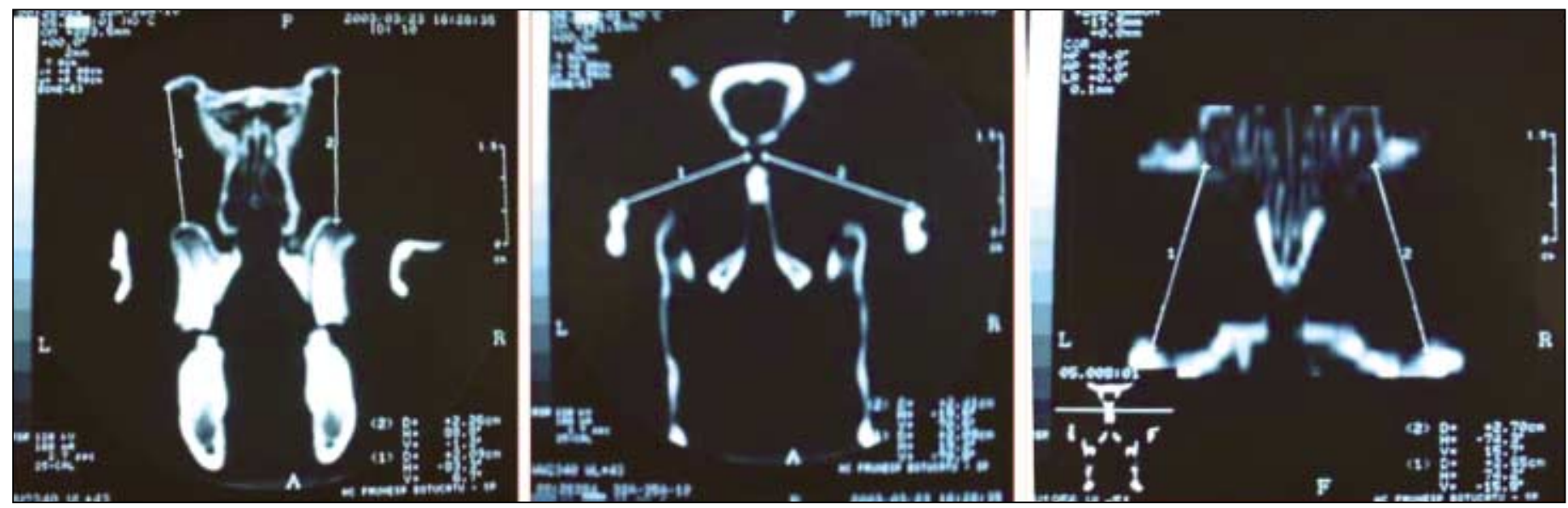

Figura 2 - Medidas relativas a órbita do coelho obtidas utilizando o método tomográfico 


$$
\mathrm{V}=4 / 3 \pi \times \mathrm{D}_{1} / 2 \times \mathrm{D}_{2} / 2 \times \mathrm{L} / 2
$$

Onde: $\mathrm{V}=$ volume; $\mathrm{D}_{1}=$ diâmetro no sentido ântero-posterior; $\mathrm{D}_{2}=$ diâmetro no sentido lateral; $\mathrm{L}=$ diâmetro maior ou longitudinal.

Usando a imagem tomográfica, foi feito também um estudo da medida da abertura orbitária, por meio do "software Image-J".

A medida volumétrica obtida pelo teste de deslocamento de água (Princípio de Arquimedes) consistiu da confecção de um molde da cavidade por impressão com Alginato (Jeltrate Chromatic - Dentsply), delimitado pelo contorno da abertura orbitária. O molde foi imerso em cilindro graduado de $100 \mathrm{ml}$ (Vidrolex) contendo água, avaliando-se o volume deslocado.

Os dados do teste de deslocamento de água foram estudados estatisticamente por análise de variância paramétrica. As outras variáveis - tipo de cirurgia, uso de implante e momento experimental - foram avaliadas por estatística não-paramétrica, para o modelo com três fatores, complementada pelo teste de comparações múltiplas simultâneas, sendo adotado como significativo o valor de $\mathrm{p}<0,05$. Foi também analisada a associação entre os métodos de medida empregados (tomografia computadorizada e teste de deslocamento de água, no caso da medida de volume; tomografia computadorizada e "software Image-J", no caso da medida da abertura orbitária), usando o coeficiente de correlação de Pearson.

\section{RESULTADOS}

Os valores obtidos pelo exame tomográfico estão apresentados nos gráficos 1 e 2 . No grupo de ENCI, não houve diferença entre M1 e M2, enquanto que no M3, os volumes das órbitas foram maiores que os demais, tanto nas órbitas evisceradas (EV), como nas sem cirurgia (GN).

Dentre os animais do ENSI, os volumes orbitários foram progressivamente maiores no decorrer dos momentos experimentais, tanto do lado EV, como do lado GN.

Dentre os animais do EVCI, o M1 foi estatisticamente menor em relação aos M2 e M3, tanto do lado EV, como no GN.

Dentre os animais do EVSI, assim como os do ENSI, os volumes orbitários foram progressivamente maiores no decorrer do tempo, tanto do lado EV, como do lado GN.

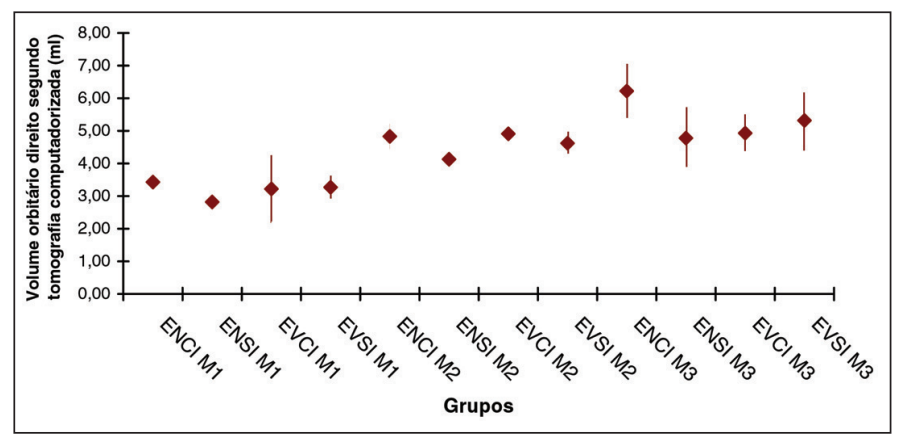

Gráfico 1 - Mediana e semi-amplitude do volume orbitário direito, medido por tomografia computadorizada

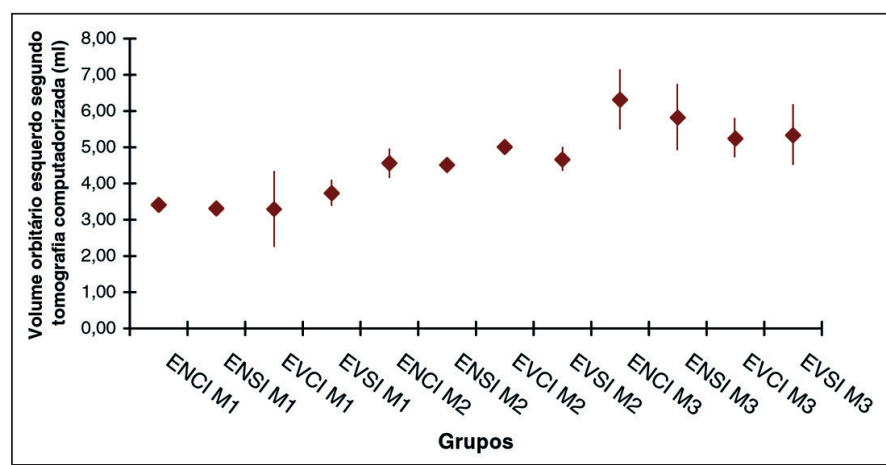

Gráfico 2 - Mediana e semi-amplitude do volume orbitário esquerdo, medido por tomografia Computadorizada

A avaliação volumétrica pelo Princípio de Arquimedes se encontra nos gráficos 3 e 4 . Observou-se aumento progressivo do volume da órbita, considerando-se os momentos de estudo. Não houve diferença estatística entre os grupos de EN e EV, com ou sem o uso do implante.

Em relação às medidas lineares de altura, largura e área da abertura orbitária, observou-se que para todas elas houve aumento progressivo nos valores absolutos, tanto na avaliação pela tomografia, como na medida pelo "software Image-J",

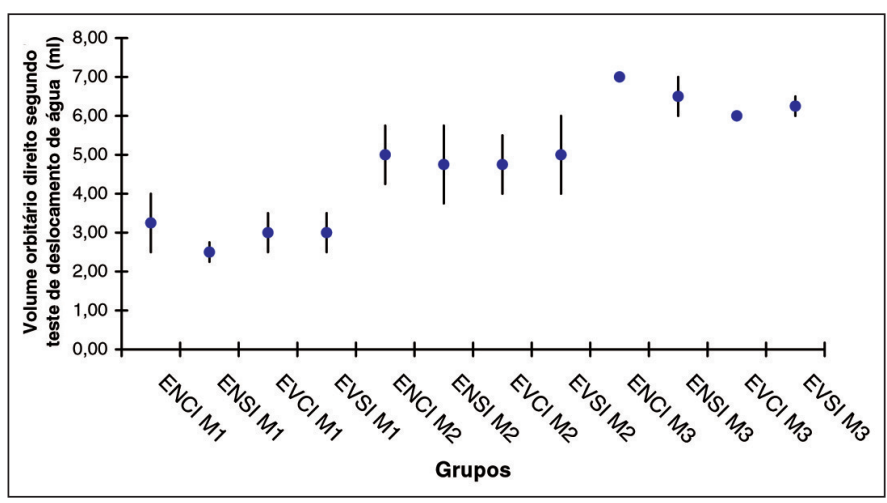

Gráfico 3 - Média e desvio-padrão do volume orbitário direito, medido por teste de deslocamento de água

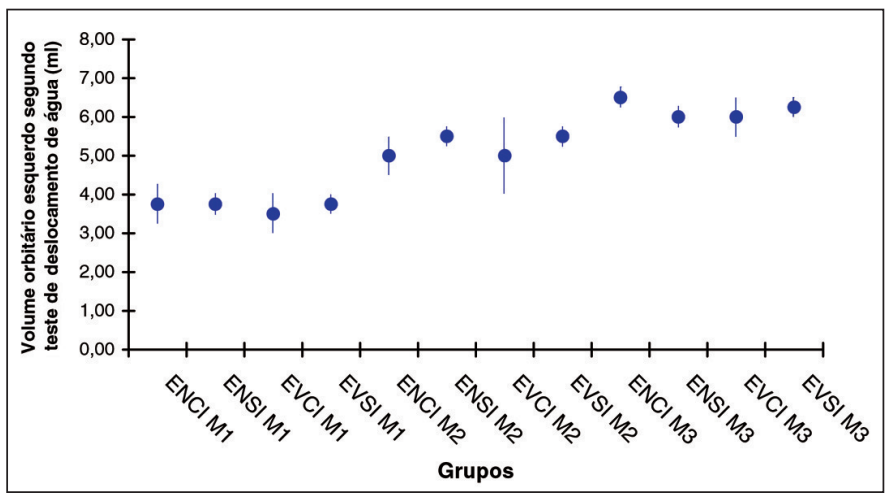

Gráfico 4 - Média e desvio-padrão do volume orbitário esquerdo, medido por teste de deslocamento de água 
ocorrendo as diferenças estatísticas de forma isolada e dispersa entre os grupos EN ou EV, no lado operado ou no lado não operado, em todos os momentos de estudo.

O teste de correlação de Pearson evidenciou associação positiva entre os métodos.

\section{DISCUSSÃO}

O coelho foi escolhido como animal modelo por apresentar rápido crescimento, órbita pequena, além de ser um animal de obtenção e manejo relativamente fáceis. Foram empregados coelhos em fase de pós-desmame, devido a aspectos éticos e a dificuldade de sobrevivência dos animais em idade mais precoce.

Neste estudo, utilizando animais com 42 dias de vida, não se obteve alteração do volume orbitário ou das dimensões da abertura orbitária estatisticamente significativas. Talvez, a idade de 42 dias seja avançada para a espécie em relação ao desenvolvimento orbitário, podendo este já ter se completado. Outros estudos utilizaram coelhos mais jovens ${ }^{(9-11)}$ tendo sido observada redução das dimensões ósseas orbitárias como profundidade, altura e comprimento em coelhos submetidos à enucleação aos 20 dias de vida ${ }^{(12)}$. $\mathrm{O}$ coelho atinge seu tamanho adulto em tempo relativamente curto ${ }^{(10)}$. Coelhos operados entre 20 e 42 dias de vida apresentam média de volume orbitário diferente de coelhos operados entre 7 e 19 dias de vida, o que corrobora com a premissa de que animais mais jovens apresentam crescimento orbitário mais rápido ${ }^{(9)}$.

Surpreendentemente, usando ou não implante intra-orbitário de $10 \mathrm{~mm}$ de diâmetro, não foram observadas diferenças no volume. Estudando coelhos enucleados entre as idades de 13 e 19 dias, concluiu-se que o implante não influenciou o crescimento orbitário ${ }^{(11)}$. Estudos em humanos mostram que, somente entre 0 a 3 anos de idade, o uso de implante afetou o crescimento da órbita ${ }^{(13)}$.

O tamanho do implante deve ser considerado. Outros estudos referem o uso de esfera de $12 \mathrm{~mm}$ ou maiores, revestidas ou não por esclera autógena ${ }^{(14-16)}$. Entretanto, optou-se no presente estudo por usar esfera de $10 \mathrm{~mm}$, uma vez que a de $12 \mathrm{~mm}$ tornaria o fechamento anterior inadequado, ainda que se preservasse a córnea.

O não encontro de diferenças entre os grupos do estudo animais enucleados e eviscerados, com ou sem o uso de implantes - poderia ser decorrente do fato da órbita de coelhos ser rasa e abrigar apenas parte do bulbo ocular. Assim, mesmo a ausência do bulbo, não levaria a alterações do tamanho da órbita.

Com relação aos métodos de medida empregados, optouse por realizar avaliações diretas, em crânio seco, uma vez que as medidas dos ossos da órbita ou os exames de imagem ${ }^{(17)}$ são mais exatos desta forma, do que quando os tecidos moles estão presentes. Os métodos de imagem permitem extrapolações in vivo das medidas diretas, feitas nos crânios $\operatorname{secos}^{(18)}$.

Neste estudo, embora tenham sido observados menores valores relativos ao volume nas cavidades anoftálmicas usando-se o exame tomográfico, esta diferença não foi estatistica- mente significativa entre os grupos, inclusive no grupo em que não foi colocado o implante.

Em estudos animais, é relatada diminuição das dimensões orbitárias, em medidas lineares, observando diminuição da profundidade de órbitas felinas de $11,5 \%{ }^{(19)}$ e também de coe$\operatorname{lhos}^{(10)}$.

Com o uso de implante retangular de PP, o volume da órbita enucleada em coelhos foi semelhante à não enucleada, observando-se que a idade em que é realizada a enucleação seria importante para definir estas diferenças ${ }^{(10)}$.

Em crânio humano com anoftalmia unilateral, observou-se em medidas lineares por radiografia, uma diminuição do tamanho de cerca de $25 \%$ da abertura orbitária, $16,7 \%$ da altura vertical, $20 \%$ da linha média à parede lateral, $12 \%$ de profundidade e $60 \%$ do volume orbital, quando comparado ao lado contralateral $^{(13)}$.

O teste de deslocamento de água apresenta desvantagens pelo fato de estar sujeito às variações da subjetividade do observador; contudo trata-se de uma técnica simples, de fácil execução e de pouco custo, permitindo uma estimativa do volume de um objeto irregular, como o é a cavidade orbitária, além de poucos riscos em danificar o material, pelo fato do molde ser facilmente retirado, graças à sua característica elástica ${ }^{(13)}$.

A avaliação do volume orbitário por meio de medidas tomográficas, pelo teste de Arquimedes, assim como pelo "software Image-J", nos mostraram semelhança de resposta, com alta correlação entre os métodos, como também relatado na literatura ${ }^{(8)}$. Esta constatação é importante, na medida que aponta para o teste do deslocamento da água como uma possibilidade muito barata e segura para a avaliação do volume orbitário em trabalhos experimentais.

A tomografia tem vantagens pela possibilidade de ser aplicado em estudos clínicos, enquanto que o teste de deslocamento de água restringe-se aos estudos experimentais ou post mortem, além de ser um método inadequado nos casos em que há phthisis ou ruptura severa do bulbo ${ }^{(19)}$.

\section{CONCLUSÃO}

Os resultados obtidos neste estudo permitem concluir que o desenvolvimento orbitário de coelhos com idade superior a 42 dias de vida não se altera quando se opta pela enucleação ou pela evisceração, ou quando se utiliza ou não implantes intra-orbitários.

O teste de deslocamento de água apresentou resultados comparáveis às medidas obtidas pela tomografia, sendo um exame de baixo custo e fácil execução, o que nos leva a sugerir a sua utilização em experimentação científica.

\section{ABSTRACT}

Purpose: To assess orbital volume in weaned rabbits, submitted to enucleation and evisceration, with orbital volume reconstitution using porous polyethylene (PP) spheres, analy- 
zed by computed tomography, by the water displacement test and external orbital measures using Image-J software. Methods: This experimental randomized study consisted of 48 albino rabbits, with 42 days of life, submitted to enucleation (EN) and evisceration (EV), with (WI) or without (NI) implantation of a 10 mm PP sphere. After 1 month (M1), 3 months (M2) and 6 months (M3), 4 animals of each group were sacrificed. The skulls were prepared for dry bone study using the maceration technique. The orbital volume was calculated by computed tomography and by the water displacement test. The results suggest that orbital development in rabbits above 42 days of life is not different regarding evisceration or enucleation, or when an orbital implant is used. The water displacement test showed similar results compared with computed tomography, making a morphometric method in experimental studies worthwhile.

Keywords: Eye enucleation; Orbit evisceration; Orbit/growth \& development; Tomography, x-ray computed; Rabbits

\section{REFERÊNCIAS}

1. Osborne D, Hadden OB, Deeming LW. Orbital growth after childhood enucleation. Am J Ophthalmol. 1974;77(5):756-9.

2. Kaltreider SA, Jacobs JL, Hughes MO. Predicting the ideal implant size before enucleation. Ophthal Plast Reconstr Surg. 1999;15(1):37-43.

3. Kaltreider SA. The ideal ocular prothesis: analysis of prosthetic volume. Ophthal Plast Reconstr Surg. 2000;16(5):388-92.

4. Kaltreider SA, Lucarelli MJ. A simple algorithm for selection of implant size for enucleation and evisceration: a prospective study. Ophthal Plast Reconstr Surg. 2002;18(5):336-41.

5. Heinz GW, Nunery WR, Cepela MA. The effect of maturation on the ability to stimulate orbital growth using tissue expanders in the anophthalmic cat orbit. Ophthal Plast Reconstr Surg. 1997;13(2):115-28.

6. Ainbinder DJ, Haik BG, Mazzoli RA. Anophthalmic socket and orbital implants. Role of CT and MR imaging. Radiol Clin North Am. 1998;36(6): 1133-47.

7. Lutzemberger L, Salvetti O. Volumetric analysis of CT orbital images. Med Biol Eng Comput. 1998;36(6):661-6.

8. Deveci M, Öztürk S, Sengezer M, Pabuscu Y. Measurement of orbital volume by a 3-dimensional software program: an experimental study. J Oral Maxillofacial Surg. 2000;58(6):645-53.

9. Sarnat BG, Shanedling PD. Orbital volume following evisceration, enucleation and exenteration in rabbits. Am J Ophthalmol. 1970;70(5):787-99.

10. Smith EM, Dryden RM, Tabin GC, Thomas D, To KW, Hofmann RJ. Comparison of effects of enucleation and orbital reconstruction using free-fat grafts, dermis grafts and porous polyethylene implants in infant rabbits. Ophthal Plast Reconstr Surg. 1998;14(6):415-24.

11. Sarnat BG, Shanedling PD. Orbital growth after evisceration or enucleation without and with implants. Acta Anat (Basel). 1972;82(4):497-511.

12. Thomson WE. The determination of the influence of the eyeball on the growth of the orbit, by experimental enucleation of one eye in young animals. Trans Ophthalmol Soc UK. 1901;21:258-68.

13. Kennedy RE. Growth retardation and volume determinations of the anophthalmic orbit. Trans Amer Ophthal Soc. 1972;70:279-97.

14. Hsu WC, Green J, Spilker MH, Rubin PA. Primary placement of a titanium motility post in a porous polyethylene orbital implant: animal model with quantitative assessment of fibrovascular ingrowth and vascular density. Ophthal Plast Reconstr Surg. 2000;16(5):370-9.

15. Rubin PAD, Popham JK, Bilyk JR, Shore JW. Comparison of fibrovascular ingrowth into hydroxiapatite and porous polyethylene orbital implants. Ophthal Plast Reconstr Surg. 1994;10(2):96-103.

16. Bigham WJ, Stanley P, Cahill Jr JM, Curran RW, Perry AC. Fibrovascular ingrowth in porous ocular implants: the effect of material composition, porosity, growth factors, and coatings. Ophthal Plast Reconstr Surg. 1999;15(5): 317-25.

17. Fountain TR, Goldberger S, Murphree AL. Orbital development after enucleation in early childhood. Ophthal Plast Reconstr Surg. 1999;15(1):32-6.

18. Kennedy RE. Effects of early enucleation on the orbit in animals and humans. Am J Ophthalmol. 1965;60:277-306.

19. Bilyk J. Enucleation, evisceration and sympathetic ophthalmia. Curr Opin Ophthalmol. 2000;11(5):372-86.

\section{CONGRESSO NACIONAL DA SOCIEDADE BRASILEIRA DE OFTALMOLOGIA}

\section{6 a 18 de Junho de 2005 \\ Centro de Convenções Ribalta Rio de Janeiro - RJ}

IN FORMAÇÕES: Sociedade Brasileira de Oftalmologia

Tel.: (21) 2557-7298 c/ Marcos

Fax: (21) 2205-2240

Email: sbo@ sboportal.org.br 